Journal of Luminescence 72-74 (1997) 368-369

\title{
Luminescence and ESR studies of photodarkening in CdS-doped glasses
}

T. Miyoshi, K. Towata, H. Matsuki, N. Matsuo

Department of Electrical and Electronic Engineering, Yamaguchi University, Tokiwadai, Ube, Yamaguchi 755, Japan

\begin{abstract}
Mechanisms of photodarkening effects have been investigated in CdS-doped glasses using time-resolved luminescence and electron spin resonance (ESR). Correlation between the ESR signal and the photodarkening effects was observed. Photoinduced centers responsible for the photodarkening are considered to be created in the interface between CdS nanocrystals and glass matrix.
\end{abstract}

Keywords: Semiconductor; Nanocrystal; Luminescence; ESR 
Semiconductor-doped glasses have large optical nonlinearity with a fast response time. Roussignol et al. [1] reported that the response time was shortened by light irradiation. This photoinduced irreversible process is often called photodarkening. The photodarkening is considered to be due to trapped electrons in glass matrix [2,3] or photoinduced centers, which act as nonradiative recombination centers $[4,5]$. Here, we report the time-resolved luminescence and ESR (electron spin resonance) spectra to investigate the mechanism of the photodarkening.

The samples investigated were CdS-doped commercial filter glasses. Toshiba Y-44, etc. These glasses were exposed to pulsed light from an $\mathrm{N}_{2}$ laser (Usho YKN-900; wavelength = $337.1 \mathrm{~nm}$, pulse duration $=5 \mathrm{~ns}$, peak intensity $=4 \mathrm{MW} / \mathrm{cm}^{2}$, repetition rate $=1 \mathrm{~Hz}$ ) at $300 \mathrm{~K}$. Time-resolved luminescence spectra were measured at $300 \mathrm{~K}$ using a short pulsed $\mathrm{N}_{2}$ laser (Laser Photonics LN120; pulse duration $=0.3 \mathrm{~ns}$ ) and an optical multichannel analyzer with gate (Princeton Instruments D/SIDA-700). The minimum gate time is 5 ns. The ESR spectra were measured at $77 \mathrm{~K}$, using an X-band spectrometer (JES FE-1X).

Main luminescence band was observed at about $440 \mathrm{~nm}$ in CdS-doped glass, Y-44. This band is attributable to the shallow-trapping state-to-band-type transition. Luminescence intensity decreases by irradiation. This result indicates that nonradiative recombination centers are created by light irradiation. These centers open an additional channel for recombination of excited carriers, so that the decay rate of luminescence may increase. Transient characteristics of luminescence were measured. The decay rate of the $440 \mathrm{~nm}$ band increases after irradiation. Although the decay rate does not recover its initial value after annealing at $200^{\circ} \mathrm{C}$, it recovers after annealing at $400^{\circ} \mathrm{C}$ for $2 \mathrm{~h}$.

Fig. 1 shows ESR spectra of glasses. ESR signals appear by light irradiation. The ESR signal near $\mathrm{g}=1.99$ disappears after annealing at $200^{\circ} \mathrm{C}$, and the signal near $\mathrm{g}=2.01$ almost disappears after annealing at $400^{\circ} \mathrm{C}$. A correlation was observed between the decay rate of luminescence and the intensity of the latter ESR signal.

We observed photoinduced signal in glass, Y-0, which does not contain CdS nanocrystals, after $\mathrm{KrF}$ laser light (wavelength $=248 \mathrm{~nm}$ ) irradiation [5]. This signal is attributable to trapped holes in glass and disappears after annealing at $200^{\circ} \mathrm{C}$.

We measured luminescence and ESR spectra of CdS-doped glass after X-ray irradiation. ESR spectrum is similar to that of $\mathrm{Y}-0$ after $\mathrm{KrF}$ laser irradiation. Although transmittance of the 
X-ray irradiated glass decreases, transient characteristics of luminescence from the glass are similar to that before X-ray irradiation. This result indicates that the trapped holes in glass matrix cause change in transmittance and do not cause change in luminescence.

Intensity of the ESR signal near $\mathrm{g}=2.01$ as a function of the size of nanocrysttals was measured [6]. The ESR signal is proportional to $r^{-1}$. This result indicates that the ESR signal is associated with the photoinduced centers at the g1ass-semiconductor interface, since the sum of the surface area of all nanocrystals is proportionttl to $r^{-1}$.

We consider the mechanism of the photodarkening. Under laser irradiation, photoinduced centers are created in the interface region between CdS nanocrystals and the glass matrix. These centers act as nonradative recombination center, so that luminescence intensity decreases and decay rate of luminescence increases.

The ESR signals almost disappear and the decay rate of luminescence recovers its initial value after annealing in $\mathrm{H}_{2}$ gas at $350^{\circ} \mathrm{C}$ for $4 \mathrm{~h}$. Since the photoinduced centers are passivated by $\mathrm{H}_{2}$, these centers are probably associated with dangling bonds.

In summary, luminescence and ESR of CdS-doped glasses are measured. Photoinduced ESR signals were observed. The correlation between the ESR signal and the decay rate of luminescence indicates that the photoinduced centers responsible for the ESR signal are associated with photodarkening. The photoinduced centers are created in the interface region between CdS nanocrystals and glass matrix. 


\section{References}

[l] P. Roussignol, D. Ricard, J. Lukasik and C. Flytzanis, J. Opt. Soc. Amer. B 4 (1987) 5.

[2] V.Ya. Grabovskis, Ya.Ya. Dzenis, A.I. Ekimov, I.A. Kudryavtsev, M.N. Tolstoi and U.T. Rogulis, Sov. Phys. Solid Stat. 31 (1989) 149.

[3] J. Malhotra, D.J. Hagan and B.G. Potter, J. Opt. Soc. Amer. B 8 (1991) 1531.

[4] B. Van Wonterghem, S.M. Saltiel, T.E. Dutton and P.M. Rentzepis, J. Appl. Phys. 66 (1989) 4935.

[5] T. Miyoshi, K. Towata and N. Matsuo, Jpn. J. Appl. Phys. 33 (1994) 6299.

[6] T. Miyoshi, H. Matsuki and N. Matsuo, Jpn. J. Appl. Phys. 34 (1995) 1837. 
Figure caption

Fig. 1. ESR spectra of glasses at $77 \mathrm{~K}$. 
Fig. 1

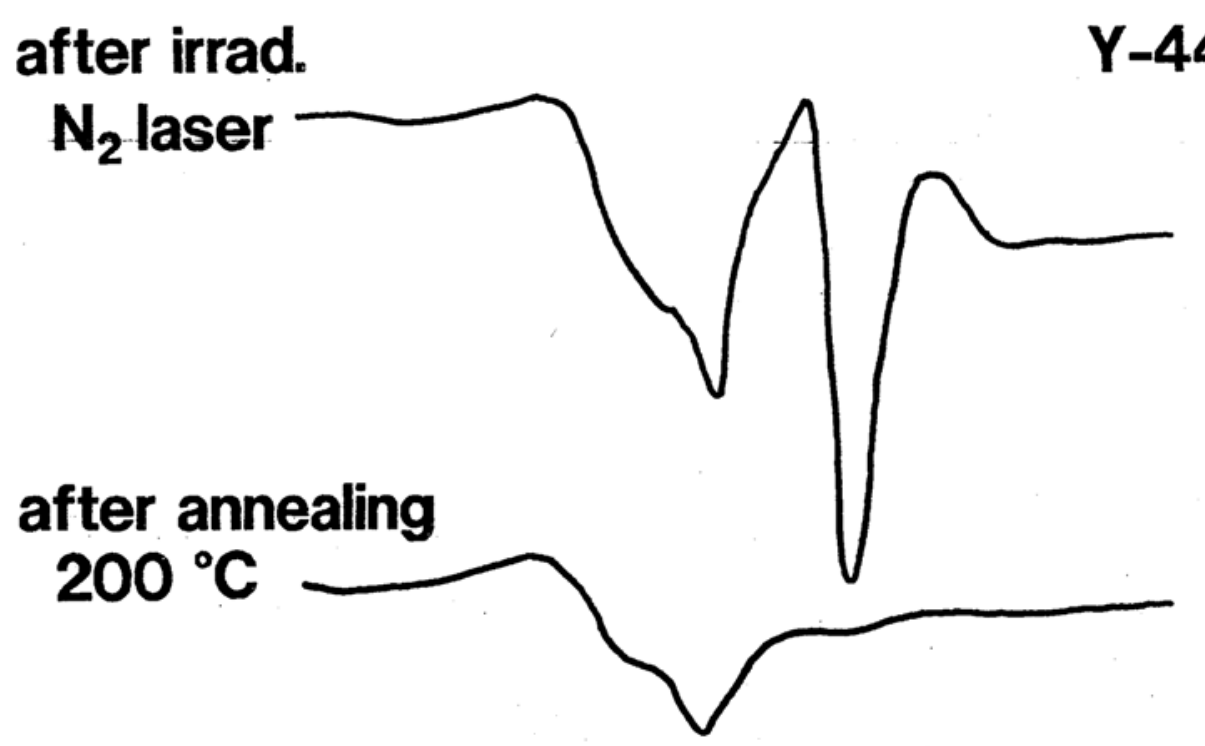

$400^{\circ} \mathrm{C}$

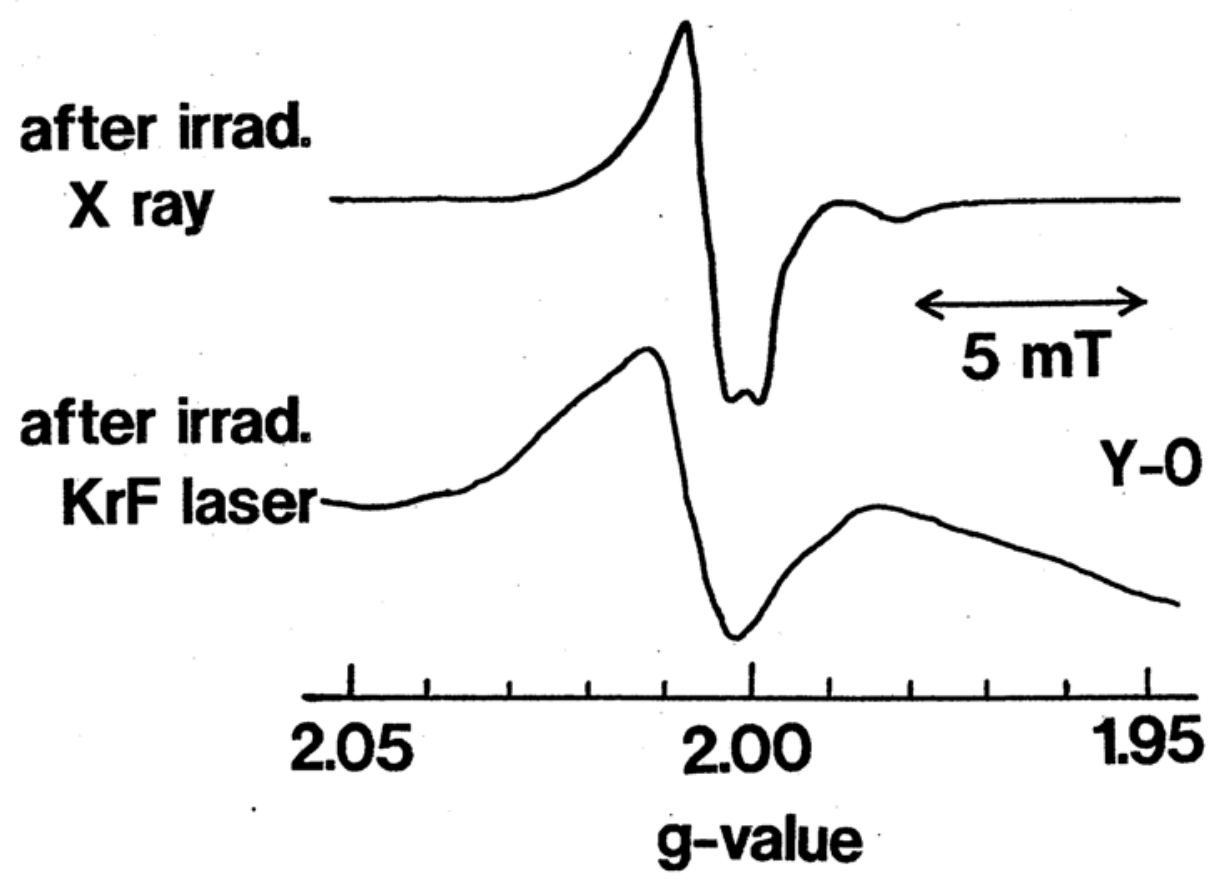

